\title{
The ESGE Junior Platform
}

\section{JP1_Case reports}

\section{JP 1_01}

\section{Single-port access laparoscopic assisted vaginal} hysterectomy in a case of uterine ventrofixation using a new reusable device

I. Arena, G. Maricosu, L. Mereu, L. Mencaglia,

G.B. Melis, S. Angioni

Department of Obstetrics and Gynaecology, University

of Cagliari and Division of Gynaecology, Villanova Hospital of Firenze, Italy

Vaginal hysterectomy may at times prove difficult and require laparoscopic assistance to ensure safety of operations. In these cases, vaginal hysterectomy has been combined with laparoscopy (laparoscopically assisted vaginal hysterectomy or $\mathrm{LAVH}$ ) to decrease morbidity in women who would otherwise have been advised to undergo abdominal surgery. We report a case in which single port laparoscopic assistance enabled vaginal hysterectomy in a patient with severe uterine ventrofixation. The use of an innovative device and tips to be applied will be described. Key-words: spal, hysterectomy, LAVH.

\section{JP 1_02}

\section{Hysteroscopic metroplasty in septate uterus}

B. Olartecoechea Linaje, D. Díaz Cobos, M. García Manero Clínica Universidad de Navarra, Spain

Congenital uterine anomalies may arise from any malformation of the Müller development process. It is reported in up to $4 \%$ of women, and is related to some cases of recurrent miscarriage and infertility. The American Fertility Society presented a classification of the congenital uterine anomalies based on the degree of failure of the development of the Müllerian ducts; anomalies are divided into seven groups:

- Müllerian hypoplasia or agenesia.

- Unicornuate uterus.

- Didelphus uterus.

- Bicornuate uterus.

- Septate uterus.

- Arcuate uterus.

- Uterine hypoplasia (secondary to DES).

Some of the anomalies may be corrected surgically, in order to improve fertility outcomes. We present the case of a 31 year old patient, nullipara, with history of mild dysmenorrhea, short menstrual cycles, and infertility (3 years). As important surgical history, she had performed a gastrc bypass when she was 28 . She attended routine gynaecological exam. She referred menarchy at 13 years old and regular cycles. Physical exploration was normal, but during ultrasound pelvic exam, we observed a septate uterus. According to her fertility desire, we performed a histeroscopy and removed the septum The resulting cavity was septum free, with normal dimensions, and we could observe both ostium tubariae.

Key-words: septate uterus, hysteroscopy, congenital uterine anomalies.

\section{JP 1 03}

Endoscopic treatment of Asherman syndrome D. Díaz Cobos, B. Olartecoechea Linaje, J.Á. Minguez Milio Clínica Universidad de Navarra, Spain 
Asherman's syndrome was first described in 1894 by Fritsch and then studied by the gynaecologist Joseph Asherman in 1948. It is characterized by the presence of adhesions and/or fibrosis within the uterine cavity caused by scars. The endometrium is composed of two layers: the internal one called basal layer, and the external functional layer, which is shed for menstruation. During performance of dilation and curettage the basal layer may be hurt, and may create adhesions or scars that could obliterate the cavity. The functional layer of the endometrium will find difficulties for regenerating, and menstrual bleeding may be blocked intrauterus, depending on the place of the adhesions. Patients usually refer changes in flow and duration of menstruation, and may feel pain or infertility. We now present one case report we treated in 2009. She was a 33 year old patient, with one previous pregnancy and eutocic delivery in 2005. In 2007 , secondary to a miscarriage, she had practiced a dilation and curettage. After this procedure, she started to present menstrual disturbances (amenorrhea and pelvic pain). The couple was looking for a further pregnancy, without success. Studied by her gynaecologist, she was diagnosed of Asherman's syndrome. She came to our hospital for a second opinion. In order to assess the damage of the endometrium, we first made an ultrasound exam, which showed a thin endometrial layer $(4 \mathrm{~mm})$, and suggested the diagnosis. We also described a corpus luteum in one of the ovaries, confirming normal ovarian function. With these findings, with the hope of being able to solve the syndrome, we proposed to do a hysteroscopy with two objectives: first, classify the level of the uterine cavity damage, and second, try to liberate the uterine adhesions. During the histeroscopy, we found a very damaged uterine cavity, with plenty of synechias, which avoided uterine distention. With microscissors, we cut the adhesions, and finally got to visualise the entire cavity, including both ostium tubariae. To avoid posterior adhesions, we introduced in the uterine cavity a Folley catheter, and filled the balloon. The patient was discharged within 24 hours with the Folley catheter and the following treatment: Amoxicillin-Clavulanic $1 \mathrm{gram} /$ 8 hours during 7 days, and daily Progyluton $(2 \mathrm{mg}$ of Estradiol and 0,5 $\mathrm{mg}$ of Norgestrel) for 21 days. After 7 days, she was asked to repeat a second series of Progyluton for 21 days. She removed the Folley catheter on the fourth day after surgery. We followed the evolution by telephone. The patient recovered her menstrual cycles and 5 months later she naturally got pregnant. Pregnancy and delivery had a normal outcome. Nowadays, she still has regular cycles. We can conclude that histeroscopy may be a successful treatment for Asherman's syndrome.
Key-words: Asherman's syndrome, hysteroscopy, Folley catheter.

\section{JP1_04}

The use of office hysteroscopy to empty a large hematometra in a 33-year-old nulliparous patient after myomectomy

J. Meyer, F. Meyer, J. Moutinho, J. Martinez de Oliveira Centro Hospitalar Cova da Beira, Portugal

Objective: To describe the successful management of a large hematometra using a continuous flow operative office hysteroscope and bipolar Versapoint electrode.

Design. Case report setting: Centro Hospitalar Cova da Beira-Covilhã, Portugal.

Patient: A 33-year-old nulliparous patient underwent a myomectomy to remove 27 large fibroids. Two months after the surgery, the patient referred to the emergency room complaining of severe pelvic pain and amenorrhea. A large hematometra was detected by transvaginal ultrasound.

Intervention(s): Hysteroscopy using vaginoscopic approach was performed in outpatient setting.

Main outcome measure(s): Resolution of the hematometra and all the clinical symptoms.

Result(s): The vaginoscopic approach allowed the treatment of the patient quickly and painless. There was no visible cervical canal. Using the three diagnostic criteria for septate uterus and a Twizzle Versapoint Electrode, a new cervical ostium was "constructed and a dark red fluid spilled out from the uterus cavity. A transvaginal ultrasound was performed 1 week later and revealed complete resolution of the hematometra.

Hysteroscopic follow-up: performed 45 days later showed a regular uterine cavity.

Conclusion(s): Vaginoscopic hysteroscopy might represent the technique of choice to threat hematometra after myomectomy in nulliparous patient.

Key-words: hematometra, myomectomy, hysteroscopy.

\section{JP 1_05}

\section{A case of haematometra following evacuation} of retained products of conception

A. Swaminathan, S. Nethra, S. Wood

Countess of Chester Hospitals NHS Foundation Trust, United Kingdom

Cervical occlusion following vacuation of retained products of conception (ERPOC) is rare. 
Case discussion: This 32 year old lady had a normal vaginal delivery after IVF. Subsequently she conceived spontaneously which resulted in missed miscarriage. She underwent ERPOC with Berkeley suction curette. Four months later she developed secondary amenorrhoea with a negative pregnancy test. She had regular cyclical period pains with no bleeding. Progesterone Challenge Test was negative. A transvaginal scan (TVS) showed an endometrium measuring $6.5 \mathrm{~mm}$ with pool of fluid in cavity consistent with haematometra. She underwent hysteroscopy and insertion of Mirena IUS. Intra-operatively minimal amount of bloody material was drained and the uterine cavity was normal. Periods became regular and she had no pain. Mirena was removed with ease with no evidence of cervical stenosis. She conceived spontaneously after that episode and went on to have a caesarean section. Seven months post delivery she developed secondary amenorrhoea with negative pregnancy test. TVS was normal. She went on to conceive spontaneously and then had an incomplete miscarriage which was managed medically.

Discussion: For Intrauterine Adhesions the ESGE adopted the classification which uses hysteroscopic findings. In obstructive amenorrhoea mechanical obstruction of the internal cervical os would lead to secondary amenorrhoea, periodic discomfort or pain, haematometra and even haematosalphinx. Hysteroscopic adhesiolysis is the treatment. Various methods have been used to stent the cervical canal including the threads of IUCD or the HSG Catheter.

Key-words: haematometra, secondary amenorrhoea, hysteroscopy.

\section{JP1_ 06}

\section{Local anaesthesia laparoscopy for Chronic Pelvic Pain} (CPP) diagnosis: a case report

T. Castellanos Alarcón, M. Moya Valdés,

T. Muñoz Fernández, A. Barrio Omeñaca, C. Gianella, M.A. Huertas Fernández

Hospital Universitario Getafe, Spain

Introduction: CPP affects the 4-20\% of the population; it involves the $10 \%$ of the visits to the primary care gynaecologist. Common causes of CPP are due to gynaecologist pathologies: endometriosis, PID, adhesions, pelvic congestive syndrome, adenomyosis, and dysmenorrhoea.

Case report: Thirty-four years old female with personal history of 4 laparotomies (3 caesareans sections and laparotomic tubal occlusion) suffers of intense cramps following postural changes, dispareunia, dysmenorrhoea and coitalgia since 2004. During emergency visits, multiple specialists (digestive experts, gynaecologist's, emergency doctors and primary care experts) with repeated laboratory tests and image controls (Rx, US, MRI) are unable to diagnose the pains cause. The patient is referred to an expert laparoscopyst in CPP. Laparoscopy under local anaesthesia discovers an empty Douglas pouch, with tense uterosacrum ligaments, uterus and adnexae clued to the parietal anterior peritoneum, due to post-surgery adhesions with omentum spreading to both iliac fossas and engulfing adnexus. Following comprehensive preoperative evaluation, it is decided to perform laparoscopic adhesiolysis surgery.

Discussion: Diagnostic laparoscopy is essential for the diagnosis, and future resolution of CPP, in much selected cases. Compared to conventional laparoscopy, local anaesthesia exploratory laparoscopy is safe and effective in the diagnosis of said pathology.

Key-words: local anaesthesia exploratory laparoscopy, Chronic Pelvic Pain (CPP), post-surgery adhesions.

\section{JP2_Complications}

\section{JP2_01}

The learning curve of total laparoscopic hysterectomy and lymphadenectomy in patients with early stage endometrial cancer: complications and clinical evolution E. Martinez Díaz, L. Aller De Pace, R. Mora Ruíz-Moyano Universitary Hospital Marqués de Valdecilla, Spain

Summary: To describe complications and clinical evolution of patients underwent total laparoscopic hysterectomy and lymphadenectomy.

Introduction: We have investigated the most common complications in a 10 cases series of total laparoscopic hysterectomy and lymphadenectomy as a learning curve.

Materials and Methods: Retrospective study. We have reviewed files of ten patients diagnosed of early stage endometrial cancer with total laparoscopic hysterectomy and lymphadenectomy .

Results: None of our patients had to be operated upon again. We did not observe any haemorrhage complications requiring another operation, but there was a case of heavy bleeding which needed blood transfusion (10\%). There was a case that we had to convert in laparoscopyassisted vaginal hysterectomy due to abdominal and pelvic adherences that complicated laparoscopic surgery $(10 \%)$. Uterine perforation was registrated in three cases 
(30\%). Postoperative complications were abdominal wall haematoma $(10 \%)$ and deep venous thrombosis $(10 \%)$. The average hospital stay was 5 days.

Discussion: Total laparoscopic hysterectomy is a feasible and safe procedure with complications rate similar to that reported in laparotomy hysterectomy.

Key-words: complications, total laparoscopic hysterectomy and lymphadenectomy, learning curve

\section{JP3 Endometriosis: Diagnosis}

\section{JP 301}

\section{Endometrioma and Deep Endometriosis (DE):} correlations in a large sample of patients

M. Dessole, E. Stochino Loi, S. Pirarba, S. Sanna, I. Arena, I. Melis, G.B. Melis, S. Angioni

Division of Gynaecology, Obstetrics and Pathophysiology of Human Reproduction, University of Cagliari, Italy

Introduction: Three types of endometriosis have been described: peritoneal, endometrioma and deep pelvic endometriosis (DE). Aim of our study was to investigate the role of endometrioma as markers of DE.

Methods: 900 consecutive surgical reports of patients with endometriosis have been collected and the association of the presence of endometrioma(s) with DE has been detailed. Age, lesions localization and pain scores were evaluated.

Results: In $50 \%$ of the patients with endometrioma, the presence of DE has been documented. Left side and the bilateral involvement were significantly more frequent in the patients with the association. In the 126 patients with endometrioma(s) but without DE the involvement of both ovaries was present only in 19,05\%. Our study showed that left side lesions are more frequent than right ones.

Discussion: The observation that left lesions tended to be more frequent and severe than right-hand ones gives some support to the idea of a different anatomical distribution of endometriotic lesions. From an anatomical point of view, these findings support the transplantation therapy in the pathogenesis of endometriosis. The moderately high positive likelihood ratios reported in this study shows that in this cohort of women with endometriosis, the presence of endometrioma could be a good indicator of deep infiltrating endometriosis in particular when both ovaries are involved and the patient presents severe pain. Patients with evidence of endometriomas should be carefully studied before surgery in order to be referred to centres that can offer the appropriate advice and treatment including the potential to manage the DE with a careful and complete laparoscopic excision of the lesions. Key-words: DIE, endoemtriosis, endometrioma

JP4_Endometriosis: Surgery

JP4_ 01

Outcomes after laparoscopic surgery for Deep Infiltrating Endometriosis: a review of 220 cases

A. Furtado Lima, P. Trompoukis, J. Nassif, I. Miranda, B. Gabriel, A. Wattiez

IRCAD, France

Study objective: To evaluate feasibility, perioperative and post-operative complications, and short-term follow-up results following laparoscopic surgery for deep infiltrating endometriosis.

Study Design: Retrospective analysis of 220 consecutive cases of laparoscopic surgery for endometriosis in 2007 to 2009 in a single-surgeon / single centre setting.

Results: Mean patient age was 32 years (range 20 to 50 years) and $70 \%$ of patients were nulliparous. Primary or secondary types of infertility were present in $49,5 \%$ of cases. Mean hospital stay was 5 days (range 1 to 13 days). The mean preoperative scores for dysmenorrhea and dyspareunia using a visual analogue scale (VAS) were 7 and 5 out of 10 , respectively. The postoperative VAS scores droped to 2 and 1 out of 10 for dysmenorrhea and dyspareunia, respectively. Whenever possible, a complete resection of endometriotic nodules was performed including bowel or bladder wall resection, partial excision, or mucosal skinning. Involvement of sigmoid, rectum and urinary tract was present in 44 (20\%), $57(25,9 \%)$ and $43(19,5 \%)$ patients, respectively. In urinary tract endometriosis, $70 \%$ concerned the bladder and $23 \%$ the right or left ureter. In $7 \%$ both, the bladder and ureter were involved. In 69 of these patients $(31,4 \%)$ a complete excision of vaginal nodule requiring vaginal removal was necessary. Ninety seven cystectomies of the left and/or right ovary were performed and 39 of these cysts $(17,7 \%)$ were over $5 \mathrm{~cm}$ in diameter. We discuss 4 intraoperative $(1,8 \%)$ and 8 postoperative $(3,6 \%)$ complications requiring re-intervention in our cohort of patients.

Conclusions: Endometriosis surgery is one of the most flagrant examples in which strategy and experience of the surgeon are determinants for a successful outcome. Our preliminary data analysis revealed an acceptable rate of intra- and postoperative complications after such a radical laparoscopic surgery approach to treat pelvic endometriosis. 
Key-words: endometriosis surgery, outcomes, retrospective study.

\section{JP4 02}

\section{Surgical endometrioma treatment with resection of posterior broad ligament}

M. Liliana, G. Carri, A. Pontis, P. Florio, L. Mencaglia

Centro Oncologico Fiorentino, Italy

Introduction: Endometrioma occurs in $17-44 \%$ of patients with endometriosis but only $1.06 \%$ of patients have exclusively ovarian involvement. In the majority of ovarian endometriomas there is an involvement of posterior broad ligament (PBL). Endometrioma and pain recurrence rate after surgery are $6.2-56 \%$ and $9.1-17.1 \%$ respectively.

Materials and Methods: We prospectively evaluate 102 consecutive patients affected by endometrioma without other evident localization of disease, who underwent to laparoscopic endometrioma excision by stripping technique and systematic PBL resection. Data on patients' characteristics, surgical and anatomo-pathological findings and follow-up were collected.

Results: 126 endometriomas were treated; in 124 of these cases there were a PBL involvement and in 87 (70.1\%) of these cases there were deep endometriosis at anatomopathological exam. After a mean follow-up of 26 months, 7 $(6.8 \%)$ endometriomas were detected at ultrasound and 19 $(18,6 \%)$ patients referred pelvic pain recurrence. 16 $(15,6 \%)$ patients without evidence of ovarian disease during the follow-up, referred a middle cycle pelvic pain.

Conclusion: Even if surgical endometrioma treatment with resection of $\mathrm{PBL}$ seems to reduce the rate of ovarian recurrence, it has to be taken in consideration the possibility of adhesion formation with subsequent Mittelschmerz syndrome.

Key-words: endometrioma, recurrence, laparoscopy.

\section{JP 4_03}

Bladder endometriosis with Trigone involvement A. Furtado Lima, P. Trompoukis, B. Gabriel, J. Nassif, A. Wattiez IRCAD, France

Summary: This video presentation demonstrates a partial cystectomy technique in a 26 years old patient, with a symptomatic bladder endometriotic nodule, localized proximal to the ureteral ostia. It is complemented with previous, intra-operatory and 6 weeks post-op cystoscopies.
Introduction: This case reports a young patient with severe endometriosis and a primary infertility. The patient was submitted to partial cystectomy.

Materials and Methods: The patient presented with a vesico-vaginal nodule of $2 \mathrm{~cm}$ with no affection of the ureters as showed in the MRI. The intervention started with a cystoscopy that revealed the precise location of the bladder nodule, proximal to the ureteral ostia. Double J catheters were inserted bilaterally. Partial cystectomy took place using the double $\mathrm{J}$ to guide this procedure, a nodule of $4,5 \times 2,5 \times$ $2 \mathrm{~cm}$ was excised. We demonstrate the technique of partial cystectomy followed by the defect correction.

Results: She was discharged with vesical catheter and double J catheters. Four weeks after the surgery, a cystography was performed excluding any leakage. The bladder catheter was removed 2 days after the examination. Double J catheter was removed 6 weeks after the surgery, under cystoscopic control. The patient is well, with improvement of her previous complains.

Discussion: The challenging location and size of this bladder nodule stresses the importance of previous insertion of double $\mathrm{J}$ catheters as well as the technique of closure in order to provide the best outcome possible in what matters to bladder size. The good results regarding symptoms improvement make this surgery the primary indication for symptomatic bladder endometriosis.

Key-words: bladder endometriosis, surgery, partial cystectomy.

\section{JP5_Hysterectomy}

\section{JP5_01}

Endometrial carcinoma staging in total laparoscopic hysterectomy and lymphadenectomy

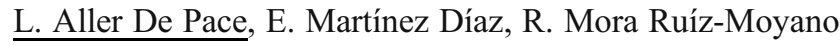
Universitary Hospital Marqués de Valdecilla Santander, Spain

Summary: To summarize the number of lymph nodes extracted in laparoscopic hysterectomy and lymphadenectomy in early stage endometrial cancer as well as complications.

Introduction: We have registrated the number of lymph nodes obteined as learning curve in total laparoscopic hysterectomy and lymphadenectomy in a 10 cases series of early pre-surgical stage endometrial carcinoma.

Materials and Methods: Retrospective study. We have evaluated ten cases of laparoscopic hysterectomy and lymphadenectomy in early pre-surgical stage of endometrial cancer. 
Results: The average number of lymph nodes of pelvic lymphadenectomy were 6.2 which $100 \%$ were negative for carcinoma. None complication was reported in lymphadenectomies. IA stage was registrated in the $22.2 \%$ of cases, IB stage was found in $33.3 \%$ of cases and finally IC stage was reported in $44.4 \%$ of cases.

Discussion: Laparoscopic lymphadenectomy is a safe procedure in patients with early stage endometrial cancer. Early stage of endometrial carrcinoma registered low rate of positive lymph nodes.

Key-words: laparascopic hysterectomy and lymphadenectomy, learning curve, lymph nodes.

\section{JP6_Imaging}

\section{JP 601}

Fast-release orodispersible tramadol as analgesia in hysterosalpingography with a metal cannula or a balloon catheter

M. De Brucker, D. Stoop, P. Haentjens, A. Talebian,

J. de Mey, P. Devroey

Vrije Universiteit Brussel-Uz Brussel, Belgium

Background: This study aimed to evaluate the potential benefit, in terms of pain relief, of the new oral fast-release orodispersible galvanic form of tramadol in women undergoing hysterosalpingography (HSG) with either a metal cannula or a balloon catheter.

Methods: In a randomized, double-blind, placebocontrolled, $2 \times 2$ factorial-design trial, conducted at a single academic centre, 128 women were assigned into groups: (I) tramadol and a metal cannula, (II) tramadol and a balloon catheter, (III) placebo and a metal cannula or (IV) placebo and a balloon catheter. The primary end-point was pain registered by the patients on $10-\mathrm{cm}$ visual analogue scales (VASs) at various times during and after the procedure. Secondary end-points included side effects and pain as assessed by the same physician during HSG.

Results: The main effect of tramadol versus placebo medication (i.e. I and II versus III and IV) was a statistically significant difference $(\mathrm{P}<0.001)$ in self-reported VAS of $-0.91(-1.35$ to -0.47$)$ on the absolute and $-33 \%(-48 \%$ to $-17 \%)$ on the relative scale in favour of tramadol. Likewise, there was a significant benefit for tramadol against placebo medication for physician-perceived VAS pain scores $(39 \%$ relative reduction; $\mathrm{P}<0.001)$. The main effect of the balloon catheter versus metal cannula (i.e. II and IV versus I and III) was a non-significant $(\mathrm{P}=0.82)$ difference in patient-reported VAS of $-0.05(-0.49$ to $+0.39)$ and $-2 \%(-21 \%$ to $+17 \%)$. There were no
medication-HSG device interactions and no differences in side effects.

Conclusions: During and after HSG, fast-release orodispersible tramadol significantly reduces pain without increasing side effects.

Key-words: hysterosalpingography, analgesia, tramadol.

\section{JP8_Innovation in Surgery}

\section{$\begin{array}{lll}\text { JP } 801 & 01\end{array}$}

Prospective study of impedance controlled system for endometrial ablation: Novasure

A. Gupta, J. Sandwell, S. Adamson, J. Goodman Maidstone District Hospital, United Kingdom

Introduction: Heavy menstrual bleeding (HMB) is an important cause of ill health accounting for $12 \%$ of all gynaecology referrals in the U.K. Novasure is a 2 nd generation ablative procedure using bipolar radiofrequency energy which has impedance control that automatically determines the depth of the tissue ablation.

Materials and Methods: The aim of our prospective study was to determine both short and long term results of Novasure endometrial ablation. Seventy eight patients were operated upon between June 2007 and Dec 2009 and followed in clinic at six months by the same clinician (JG). Further telephone consultation was made between 12 and 36 months. Results : Mean patients age undergoing ablation was 46.6 years. Sixty six (85\%) patients had associated dysmenorrhea with mean VAS of 6.81 . Thirty one patients (40\%) underwent Novasure under GA, 37(47\%) under sedation and $10(13 \%)$ under LA. No major complications were noted. Vaginal bleeding lasted for median of seven day. Median requirement of analgesia was for two days.

At six months follow up: $50 \%$ patients were amenorrhoeic and overall $93.5 \%$ had improvement of HMB. Fifty two (67\%) patients had no dysmenorrhoea at six months. Mean VAS of dysmenorrhoea was 3.2. Seventy patients $(90 \%)$ were satisfied. Five patients underwent second surgery.

Long term follow up: Mean 19 months ( $n=36$ ): $47 \%$ were amenorrhoeic and overall 97\% had improvement of HMB. Thirty four (94\%) patients were satisfied.

Discussion: Our study suggests that Novasure is a very effective treatment of HMB in both the long and short term. Post operative analgesia requirement was minimal with high satisfaction rate (90-94\%). Novasure is an effective, day case procedure for treating HMB with low complication rate and high patient satisfaction.

Key-words: bipolar radio-frequency energy, heavy menstrual bleeding, patient satisfaction 
JP9 Myomectomy

JP9_01

Laparoscopic excision of intraligamentary leiomyoma with deep retroperitoneal involvement

S. Aniorte, A. Sanroma, R. Romaguera, J. Gilabert-Estelles, A. Pellicer

Department of Gynaecology, Universitary Hospital La Fe, Valencia, Spain

We report a case of a 54-year-old multiparous woman, with a voluminous intraligamentary myoma with deep involvement of the left paravesical fossa. Doppler ultrasound showed a high-flow vascular pattern at the intramural portion of the myoma. The myoma volume has remained stable during the last 12 months and the patient consulted for increasing hypogastric strain in the last 3 months. A laparoscopic hysterectomy with bilateral salpingoophorectomy was indicated. After the establishment of the pneumoperitoneum, we observed a $9 \mathrm{~cm}$ cerebroid parauterine mass with aberrant vascularisation, which deeply infiltrated the paravesical and pararectal fossa with lateral extension up to the iliac vessels. A systematic dissection of all the retroperitoneal structures was performed and the ureter was carefully separated from the mass laterally. An aberrant vascular venous network, which drained to the hypogastric vein, was identified at and ligated. Uterine artery was clipped at its origin, facilitating the hysterectomy procedure. Vaginal extraction of the specimen was possible without morcellation, due to the soft consistency of the tissue. An intraoperative frozen section of the specimen gave the diagnosis of involutive leiomyoma without malignant cells. After the closure of the vagina, the bladder was filled with $200 \mathrm{ml}$ of methylene blue which showed a small area of leakage that was uneventfully repaired using a double layer of intracorporeal suturing. The patient was discharged uneventfully 72 hours after and the bladder catheter was maintained for 7 days.

\section{JP10_Office \& Diagnostic Hysteroscopy}

\section{JP 10_01}

Does cervical preparation before outpatient hysteroscopy reduce patients' pain experience? A systematic review

N.A.M. Cooper, P.S. Smith, K.S. Khan, T.J. Clark

University of Birmingham, BirminghamWomen's Hospital, United Kingdom
Objective: To compare the effect on pain and need for cervical dilatation of various methods of cervical preparation before outpatient hysteroscopy.

Design: Systematic review and meta-analysis of randomised controlled trials (RCT's). Setting: Outpatient hysteroscopy clinics.

Participants: Women undergoing diagnostic or operative hysteroscopy in the outpatient setting (i.e. without general anaesthesia).

Study selection criteria: Medline, Embase, CINAHL, the Cochrane library and reference lists of relevant studies. Two reviewers independently selected trials which assessed the effect on pain when cervical preparation was given prior to outpatient hysteroscopy. Data were also extracted on the effect on dilatation, side effects, trauma and feasibility

Results: Six studies were included in the systematic review. Data regarding pain and the need for cervical dilatation were unsuitable for meta-analysis. Although a single study suggests that there may be a benefit for postmenopausal women, there is no high quality evidence that giving misoprostol prior to outpatient hysteroscopy reduces the pain experienced by women of reproductive age. There is some evidence that prostaglandins reduce the force and requirement for dilatation of the cervix beyond $5 \mathrm{~mm}$. Mifepristone does not alter the pain experience or incidence of dilatation of the cervix if given to premenopausal women prior to outpatient hysteroscopy.

Conclusions: There is no evidence to recommend the routine administration of mifepristone or misoprostol to patients before outpatient hysteroscopy. Cervical priming with vaginal prostaglandins may be considered in postmenopausal women if using hysteroscopic systems greater than $5 \mathrm{~mm}$ in diameter.

Key-words: outpatient hysteroscopy, prostaglandins, cervical preparation.

\section{JP 10_02}

Endocervicoscopy: a new technique for the diagnostic work-up of cervical intraepithelial neoplasia allowing a tailored excisional therapy in young fertile women G. Bifulco, R. Piccoli, G. Lavitola, A. Di Spiezio Sardo, M. Spinelli, A. Cavallaro, C. Nappi

Department of Gynaecology and Obstetrics, and Pathophysiology of Human Reproduction, University of Naples Federico II, Italy

Study objective: To propose and validate endocervicoscopy, a new technique for patients with high-grade squamous intraepithelial lesion (H-SIL) and unsatisfactory or negative colposcopy. 
Materials and Methods: From November 2006 to February 2008, 95 consecutive patients (mean age: 32.7 years) with repeatedly positive cervicovaginal cytology for highgrade squamous intraepithelial lesion (H-SIL) and negative $(n=23)$ or unsatisfactory (i.e. non-visible squamo-columnar junction) $(n=72)$ colposcopy, were enrolled. Endoscopic evaluation of the endocervical mucosa was performed with an office continuous-flow hysteroscope after application of acetic acid 5\% (endocervicoscopy). Patient diagnosed with abnormal cervical findings (A group) underwent targeted biopsies of the visualized abnormalities. Negative patients at endocervicoscopy (B group) underwent blind curettage of endocervix. Eventually, both groups underwent a cold knife conization. Sensitivity, specificity, accuracy, positive predictive value (PPV) and negative predictive value (NPV) of endocervicoscopy plus targeted biopsy and curettage, compared to the reference test, represented by surgical specimen of cervical conization.

Results: Endocervicoscopy plus targeted biopsy showed significantly higher sensitivity than cervical curettage. $(79 \%$ vs $66 \% \mathrm{p}<0.05)$ and comparable specificity $(100 \%$ vs $100 \%$ ), diagnostic accuracy ( $80 \%$ vs $76 \%$ ) and PPV (99\% vs $100 \%$ ). Both technique had low NPV (endocervicoscopy: $51 \%$; cervical curettage: $54 \%$ ) due to the high rate of false negatives.

Conclusions: Endocervicoscopy appears to be a safe and effective office technique improving the diagnostic work-up of cervical intraepithelial lesions. The precise localization of the lesions allows for the depth of cone excision to be tailored, thus leading to a more conservative treatment and preserving the future fertility of women.

Key-words: cervical Intraepithelial neoplasia, conization, endocervicoscopy.

\section{JP 10_03}

Nerve fibres in endometrium: do they play a role in generating pain during office hysteroscopy ?

A. Di Spiezio Sardo, L.M. Sosa Fernandez, M. Spinelli, M. Guida, B. Zizolfi, M. Lisbino, C. Nappi

Department of Gynaecology and Obstetrics,

and Pathophysiology of Human Reproduction, University of Naples Federico II, Italy

Summary: A Prospective trial was conducted to investigate the presence and role of nerve fibres in the functional layer of endometrium, supporting the hypothesis that endometrial nerve fibres may play a role in pain generation during endometrial biopsy at office hysteroscopy.

Introduction: A Prospective trial (Canadian Task Force II2) was conducted to investigate whether nerve fibres are present in the functional layer of endometrium and whether they may play a role in generating pain during office hysteroscopy with endometrial biopsy.

Materials and Methods: 99 women underwent office hysteroscopy with eye-targeted endometrial biopsy. Office hysteroscopy performed with vaginoscopic approach without any analgesia or anesthesia. Visual analogue scale (VAS) pain score was assessed throughout the following steps of the procedure [(1) vaginoscopy, (2) inspection of the uterine cavity, (3) endometrial biopsy]. Positivity for S100, NSE (neuronal markers) and specific neuropeptides (VIP, NPY, NKA, NKR1) was evaluated by immunohistochemical analysis on specimens retrieved by eye-targeted biopsies.

Results: Patients with a VAS pain score $>2$ during endometrial biopsy (group $A=28$ ) showed significantly higher values of S100, NSE, VIP, NPY, NKA, NKR1 immuno-active nerve fibres in comparison with patients with a VAS score $<2$ (group $\mathrm{B}=71)(\mathrm{P}<.05)$. The proportion of women diagnosed with endometriosis/adenomyosis or myomas was significantly higher in group $\mathrm{A}$ in comparison with group $\mathrm{B}(14 / 28$ vs $18 / 71 \mathrm{P}=0.018)$.

Discussion: The results of the index study seem to support the hypothesis that immuno-active nerve fibres running through the functional layer of the endometrium may play a role in pain generation during endometrial biopsy at office hysteroscopy. However the origin of such fibres. as well as the likely association of nerve fibers with some gynaecological pathologies (i.e. endometriosis, adenomyosis, uterine myomas) needs to be further investigated.

Key-words: pain, nerve fibres, hysteroscopy.

\section{JP10_04}

Hysteroscopy used in endouterine infertility. A clinical study of 105 patients

N. Miron, D. Socolov, I. Anghelache-Lupascu, R. Socolov, C. David, S. Butureanu

University of Medicine Gr. T. Popa Lasi, Romania

Our study seeks to examine both the frequency and types of endouterine pathology involved in female infertility (intrauterine adhesions, fibroids, polyps, endometritis, adenomyosis).

Materials and Methods: The study was conducted on 105 infertile patients admitted in a gynaecologic hospital of IasiRomania, between January 2008-January 2010. All patients were examined by hysteroscopy combined with laparoscopy for an infertility investigation protocol under general anesthesia.

Results: We investigated patients aged between $22-41$ years (mean 31.5 years) with primary infertility-40 cases 
(38.1\%) and secondary infertility-65 cases (61.9\%). There was no recorded incident or complication. In 31 cases $(29.52 \%)$ the hysteroscopic aspect was normal (other causes of infertility) and in 74 cases $(70.48 \%)$ it revealed various pathologies, as follows:

- Intrauterine adhesions-35 cases (33.3\%)

- Polyps-18 cases (17.14\%)

- Endometritis-10 cases $(9.52 \%)$ certified by anatomopathological examination

- Hypotrophy / atrophy of the endometrium-31 cases $(29.52 \%)$

- discrepancy with menstrual cycle phase

Conclusions: Hysteroscopy proved to be a useful and relatively "easy to perform" maneuver in the diagnosis and treatment of endouterine pathology. It is important to note that one can identify diseases not diagnosed by ultrasound or hysterosalpingography (endometritis and filmy adhesions).

Key-words: hysteroscopy, infertility, endouterine pathology.

\section{JP10_05}

Office operative hysteroscopy. Results on pain, diagnosis and treatment of uterine pathologies

S. Sanna, E. Stochino Loi, S. Pirarba, I. Arena, G. Maricosu, G.B. Melis, S. Angioni

Division of Gynaecology, Obstetrics and Pathophysiology of Human Reproduction, University of Cagliari, Italy

Introduction: Office operative hysteroscopy is a recent technique that enables treatment of uterine pathologic disorders in the ambulatory without sedation. Aim of our study was the evaluation of this approach in our patients.

Materials and Methods: 120 consecutive patients underwent office operative hysteroscopy for the following indications: abnormal uterine bleeding, infertility and abnormal endometrial pattern at transvaginal ultrasound. Vaginoscopic approach using saline as distension media and the Bettocchi hysteroscope (Karl Storz, Germany) or Versascope (Gynecare, USA) were used in all procedures. Cold scissors, graspers and bipolar electrodes (Twizzle, Versapoint, Gynecare, USA) were employed. Pain was measured during the different phases of the examinations.

Results and Discussion: The diagnostic procedures were successfully completed in 118 patients and the correlation with the pathology was very high. Two postmenopausal women presented an important stenosis that did not consent the introduction of the instrument without sedation. The operative procedure was completed in $90 \%$ of the patients. Patient's acceptability and satisfaction were very high.
Discussion: The office operative hysteroscopy has great potential of development in the treatment of uterine pathology. The use of modern instrumentation and correct technique consent to avoid the treatment under of the majority of the cases avoiding anaesthesia and reducing the waiting list.

\section{JP11_Oncology}

\section{JP11 01}

VEGF mRNA is increased in metastatic endometrial carcinoma compared to non-metastatic endometrial carcinoma

G. Brunnmayr, B. McKinnon, M. Körner, A. Kappeler, A. Günthert, B. Jahns, N.A. Bersinger, M.D. Müller Department of Obstetrics and Gynaecology, Bern, Switzerland

Introduction: Routine lymphadenectomy for the treatment of endometrial carcinoma is still considered controversial. Identifying primary tumors at risk for metastasis prior to surgical staging could reduce unnecessary lymphadenectomies. To identify specific markers that indicate metastasic potential we have analysed metastatic and non-metastatic endometrial carcinomas. The mRNA levels of vascular endothelial growth factor (VEGF) and phosphatase of regenerating liver 3 (PRL3), the protein expression of estrogen receptor $\alpha$ and $\beta$ (ER- $\alpha)(E R-\beta)$ and progesterone receptor (PR) and the association between them was determined.

Materials and Methods: Patient records were searched for women that underwent a complete surgical staging for endometrial carcinoma. Patients were split into two groups, metastatic and non-metastatic, and quantitative polymerase chain reaction (qPCR) for VEGF and PRL3 and immunohistochemistry for ER- $\alpha$, ER- $\beta$ and PR performed on samples from each patient.

Results: VEGF mRNA was significantly higher in metastatic compared to non-metastatic samples $(+\mathrm{ve}=1.97 \pm 0.27$, $-\mathrm{ve}=$ $1.19 \pm 0.21 ; \mathrm{p}=0.0455)$. A significant negative correlation between VEGF mRNA and ER- $\alpha(r=-0.6931, n=9, p=$ 0.0385 ) in metastatic samples, and a significant positive correlation between VEGF mRNA and PR $(r=0.4952 \mathrm{n}=17$, $\mathrm{p}=0.0432)$ in non-metastatic samples was observed. PRL3 expression was significantly negatively correlated $(\mathrm{r}=$ $-0.4342, \mathrm{n}=26, \mathrm{p}=0.0435$ ) with $\mathrm{ER}-\alpha$ expression in all samples. Discussion VEGF mRNA expression may represent a potential biomarker for metastatic endometrial cancer. VEGF expression is associated with ER- $\alpha$ in metastatic tissue and PR in non-metastatic tissue.

Key-words: endometrial cancer, lymphonodectomy, gene expression. 


\section{JP11 02}

Fertility-sparing combined hysteroscopic and medical treatment of atypical endometrial lesions in young women

G. Bifulco, A. Di Spiezio Sardo, N. De Rosa, A. Bertrando, G. Lavitola, M.L. Spinelli, I. Morra, C. Coppola, C. Nappi Department of Gynaecology and Obstetrics, and Pathophysiology of Human Reproduction, University of Naples Federico II, Italy

Background and aims: To assess safety and effectiveness of combined hysteroscopic and medical treatment of atypical endometrial lesions in young women to preserve their fertility.

Methods: In the Department of Gynaecology and Obstetrics of "Federico II" University of Naples 14 patients (mean age $32.6 \pm 3.5 \mathrm{yrs}$ ) with a diagnosis of atypical polypoid adenomyoma (APA) $(n=4)$, atypical endometrial hyperplasia (AEH) $(n=6)$ and well differentiated (G1) endometrial cancer (EC) $(n=4)$ were enrolled in a prospective study. For all patients were excluded cancer invasion and metastases. All patients were treated with hysteroscopic resection of endometrial atypical lesions preserving the basal endometrial layer. Furthermore a levonorgestrel releasing intrauterine system (LNG-IUS) was inserted in all patients. GnRH analogues for 6 months were also administered to 2 of those 4 patients with a pretreatment diagnosis of EC.

Results and Conclusions: At 24 months of follow-up no patient had a progression of the endometrial disease. Ten patients had a complete remission (CR) with normal functional endometrium. Two patients had a partial response (i.e. a regression of endometrial carcinoma to atypical hyperplasia). No recurrence was detected at the follow-up. Four out of the 10 patients with a diagnosis of CR had a pregnancy after LNG-IUS removal and 6 patients decided to continue contraceptive LNG-IUS treatment. Hysteroscopic resection of endometrial atypical lesions preserving the basal endometrial layer, followed by medical treatment, is a safe and effective treatment, in young women desiring to preserve their fertility.

Key-words: atypical endometrial hyperplasia, conservative treatment, hysteroscopy.

\section{JP11_03}

Lymphatic vessels occlusion evaluation by different methods of energy delivery: an histologic study

M. Gabbanini, E. Solima, F. Martinelli, B. Valeri,

B. Grijuela, F. Hanozet, A. Ditto, F. Raspagliesi
Gynaecology Oncology, National Cancer Institute, Milan, Italy

Summary: This study evaluates thermal and mechanical effects on lymphatic vessels after applying different types of energy on lymphatic stripes removed after pelvic and paraortic laparoscopic lymphadenectomy. This study confirms the reduction of the lymphatic vessels size however not always a complete reduction of lumen size is obtained. Introduction: Lymphorrhea and lymphocysts formation are one of the most common complications of paraortic and pelvic lymphadenectomy. To reduce operative times and to accomplish an adequate hemostasis several devices have been introduced for laparoscopic use. We compared the thermal and mechanical effects on lymphatic vessels after applying energy on lymphatic stripes removed after pelvic and paraortic laparoscopic lymphadenectomy.

Materials and Methods: In ten consecutive cases of laparoscopic pelvic and paraortic lymphadenectomy, we cauterized lymphatic tissue with the use of Ligasure (Valleylab, Boulder Co), Enseal (Ethicon Endo-Surgery, Cincinnati, Ohio), Ultracision (Ethicon Endo-Surgery, Cincinnati, Ohio), Biclamp (Erbe, Tübingen, Germany). Tissue was fixed in $10 \%$ buffered formalin and embedded in paraffin; $4 \mathrm{~m}$ thick paraffin sections were stained with haematoxylin and eosin. Immunostaining with CD31 antibody was performed. Histological sections have been evaluated by three different pathologists. The lumen size reduction and the rate of reduced size was evaluated and scored.

Results: All the laparoscopic devices create a significant reduction of the lymphatic vessels size after the application of energy. This effects however are not complete in more than $80 \%$ of application. No statistically significant differences where found between the different devices used.

Discussion: The use of different energies reduces the lymphatic vessels size and the risk of lymphorrhea and lymphocyst formation, however not always a complete reduction of lumen size is obtained.

Key-words: laparoscopy, lymphadenectomy, thermal and mechanical effects.

\section{JP 1104}

Hysteroscopy and endometrial precancerous and cancerous lesions

A. Popov, E. Golovina

Moscow Region Research Institute Obs $\mid$ Gyn, Russia

Aims: The objective of this study was to evaluate the incidence of atypical endometrial hyperplasia and adenocarcinoma of 
endometrium encountered during routine hysteroscopic and resectoscopic surgery in women with AUB.

Methods: Retrospective analysis of 800 women (mean age 62,5 years, range 33-79) with AUB referred to the Dp. Endoscopy in 2007-2009. Hysteroscopy were performed in 600 women (I group), resectoscopy surgery were performed in 200 women (II group). Endometrial histology was taken as the final diagnosis. Visial diagnoses of endometrial adenocarcinoma in I and II group were compared with the histopatological results of endometrium specimen.

Results: Hysteroscopic biopsy was full in 800 patients. No complication related to hysteroscopy and resectoscopy was observed. The preoperative tumor grade by hysteroscopy correlated with the final grade in $97,1 \%$ of cases. In I group there were $44(7,3 \%)$ cases of atypical endometrial hyperplasia and $18(3 \%)$ cases of endometrial cancer. In II group $-10(5 \%)$ cases of atypical endometrial hyperplasia and 3 $(1,5 \%)$ cases of endometrial cancer. In 21 cases that underwent radical surgery, no cancerous cells in peritoneal washings were found. In combination with the endometrial biopsy, sensitivity of hysteroscopy was $100 \%$ for the detection of hyperplasia or cancer.

Conclusions: We suggest that hystero-resectoscopy does not increase the risk of dissemination of malignant cells within the peritoneal cavity and does not worsen the prognosis of the disease. The samples taken under visual control provide more precise and earlier diagnostics of endometrial cancer. We could considered hysteroresectoscopy, primarily as a full diagnostic procedure.

Key-words: endometrial cancer, hysteroscopy, dissemination of malignant cells.

\section{JP11_05}

Inclusion of diagnostic laparoscopy in the advanced ovarian cancer work algorithm

M. Gurrea, S. Fuster, E. Rodriguez, F. Ruiz, S. Domingo, A. Pellicer

La Fe University Hospital, Valencia, Spain

Summary: One of the main prognostic factors in ovarian cancer management, especially in the advanced stages, is the achievement of complete optimal surgery. Identifying patients who will benefit of a previous neoadjuvant chemotherapy improves posterior results.

Objective: To evaluate laparoscopy as a diagnostic procedure in the election of treatment for advanced ovarian cancer (IIIC stage).

Materials and Methods: During the period 2009-2010 a diagnostic laparoscopy was done to those patients with an ovarian cancer IIIC stage suspect due to image study. In that laparoscopy a revision of the entire abdominal cavity, ascitis aspiration and a tumour biopsy were performed; always with an accessory $5 \mathrm{~mm}$ trocar in medium line. With the information obtained through the laparoscopy and a previous thorax-abdomen CAT, debulking primary surgery or primary neoadjuvancy was decided. It was evaluated: procedure morbility, primary optimal surgery rate and interval optimal surgery rate.

Results: 21 women with advanced ovarian cancer suspect were included, performing in 15 of them a previous explorative laparoscopy. 11 patients underwent a primary ovarian cancer surgery, being 9 of them optimal surgeries (81\%) with 5 intestinal resections cases and 3 diaphragmatic cupula resection cases. The 2 other cases were suboptimal surgeries, 1 of them with no previous laparoscopy. 6 patients were differed for interval surgery after 3 cycles of neoadjuvant chemotherapy with carbotaxol. Of these, 5 cases were optimal surgeries (83\%) (1 intestinal resection and 1 diaphragmatic cupula resection). Global rate of optimal surgery was $82 \%$.

Conclusions: The inclusion of laparoscopy as a tool in the management of advanced ovarian cancer is an effective meassure, since it allows the therapeutical orientation towards primary surgery or neoadjuvant chemotherapy with good posterior results.

Key-words: advanced ovarian cancer, optimal surgery, laparoscopis management.

\section{JP 11_06}

Follow up of patients with borderline ovarian cancer; comparison of laparoscopic and laparotomic treatment outcomes

M. Jakimovska, B. Kobal, S. Leposa

University Clinical Centre Ljubljana, Slovenia

Background: Laparoscopy has only recently been introduced as therapeutic surgical approach in patients with borderline ovarian cancer.

Materials and Patients: We present retrospectve analysis of 111 patients with borderline ovarian cancer surgically treated by laparoscopy or laparotomy in the period from 2000 to 2009. Preoperative clinical data, (ultrasound characteristics and level of $\mathrm{Ca} 125$ ), intraoperative procedures and postoperative follow up were collected from medical records and analized.

Results: 53 patients were surgically treated by laparoscopy and 58 by laparatomy. From laparoscopy treated patients in 10 cases there was a conversion to laparotomy for final surgical treatment, while in other 43 laparoscopic procedures were presumed as definitive primary treatment. All procedures performed at laparotomy were presumed as 
definitive. No patient had any adjuvant treatment. $93 \%$ of the patients, attended regular control visits twice a year for 3 years period, where ultrasound and Ca125 was controlled. During the follow-up 18 of the patients treated with laparoscopy had a second look operation: 10 had second look laparoscopy and 8 laparotomy, there were two relapses confirmed. Conclusion: Laparoscopy is a treatment of choice in patients with borderline ovarian cancer and according to our results the outcome is comparable to laparotomy treatment outcomes.

Key-words: borderline, ovarian cancer, follow-up.

\section{JP11 07}

\section{Complete cytoreduction of recurrent ovarian malignancy using neutral argon plasma}

T.K. Madhuri, A. Tailor, S.A. Butler-Manuel

Royal Surrey County Hospital, United Kingdom

Background: "Optimal cytoreduction" $(</=1 \mathrm{~cm})$ is associated with increased survival and disease free interval for EOC with best results following complete resection. PlasmaJet ${ }^{\mathrm{TM}}(\mathrm{PJ})$, a new device producing a jet of argon plasma by heating argon gas may be used to vaporise small tumour nodules. Aim Evaluate the feasibility and outcome of conversion from optimal cytoreduction of recurrent disease $(</=1 \mathrm{~cm})$ to microscopic disease only in laparoscopic surgery.

Materials and Methods: Prospective study in tertiary oncology centre. PJ used in 6 laparoscopic debulking procedures. Patient demographics, intra and post-operative data collected. Size/location of pre-surgical disease, procedures performed, tissue and anatomical location subjected to $\mathrm{PJ}$, power settings and time taken to ablate tumour deposits recorded.

Results: All 6 presented with recurrence of Stage IIIC EOC. 2/6 presented with recurrent disease $>19$ months following treatment. PJ used to treat peritoneal deposits of EOC metastases on serosal surface of small and large bowel in all 8 cases and treat deposits on the serosal surface of the liver, diaphragm and peritoneal undersurface of the pericardium with no adverse affects. Laparoscopic ablation of diaphragmatic deposits, pericardial pericardium carried out using PJ in 3 cases. Disease on liver surface and diaphragmatic nodules resected with no visible disease.

Conclusions: Preliminary data on feasibility and safety are reported suggesting that $\mathrm{PJ}$ is an innovative surgical device with several features well suited for the destruction of EOC implants and tumour plaques.
Key-words: epithelial ovarian cancer, plasmaJet, optimal cytoreduction

\section{JP11_08}

Ovarian cysts: when to observe, when to intervene, when to refer

R.D. Meneni, M. George

University Hospital of North Tees, United Kingdom

Introduction: Ovarian cysts are common. The aim of clinical management is to optimize the treatment of malignant and premalignant cysts while minimising intervention for cysts likely to resolve spontaneously. Multiple studies have shown that the outcome of ovarian cancer is better if initial treatment was managed by gynaecologists than generalists. The dilemma then is when to observe, when to intervene, and when to refer.

Aim: The aim of this study is to compare actual practice with optimal practice for management of ovarian cysts.

Materials and Methods: A retrospective case note review of 175 women with ovarian cysts over $40 \mathrm{~mm}$ was done. Patients were classified into low RMI $(<200)$ and high RMI ( $>200)$ based on CA125, menopausal status and ultrasound features. Low RMI: 66\% were premenopausal, $34 \%$ postmenopausal. Most common presentation was pain in $53.3 \%$ of cases, mass abdomen in $20 \%$ and an incidental finding in $26.6 \%$. In the post menopausal group, $98 \%$ of the women with simple cysts less than $5 \mathrm{~cm}$ had conservative management. Surgery was opted for in women with cyst size larger than $5 \mathrm{~cm}$ and RMI $>25$. All the post menopausal women had bilateral salphingooophorectomy. Histology revealed cancer in 3 cases. In the premenopausal group, women with the cyst size less than $6 \mathrm{~cm}$ predominantly had conservative management (72\%).With larger cysts $>6 \mathrm{~cm}$, surgery was the common method of treatment. One had the cyst aspirated, six had cystectomy and the rest had oopherectomy. Laparotomy was opted with a mean cyst size $>10 \mathrm{~cm}(9 \mathrm{~cm}-17 \mathrm{~cm})$. Laparoscopy was commonly adopted with cyst size less than $9 \mathrm{~cm}$. In the conservative management group, $46 \%$ of the cases the cyst regressed and rest the cyst persisted. Histology revealed borderline tumour in 3 cases and the rest had benign pathology. High RMI: $40 \%$ were premenopausal and $60 \%$ postmenopausal. Most common presenting symptom was pain in $48 \%$, incidental finding of mass in $11 \%$. In the postmenopausal group, $100 \%$ of the cases were referred to the tertiary centre as per guidelines. $81 \%$ of these women underwent primary surgery and rest had chemotherapy followed by delayed debulking. Histology 
revealed malignancy in $77 \%$ of the women; hence the referral pathway had $77 \%$ positive predictive value. In the premenopausal group, $45 \%$ had surgery, $55 \%$ were managed conservatively. Of all the patients who had surgery, $60 \%$ of the patients were referred to tertiary centre and the rest operated at North Tees. Histology revealed borderline ovarian tumour in 1 patient and rest had benign pathology.

Conclusions: Each unit should have guidelines to standardize the management of ovarian cysts. In asymptomatic simple cysts conservative management is reasonable as upto $50 \%$ regress in 6 months. In low risk cases laparoscopy is the ideal modality of management. In postmenopausal women, ovarian cysts with RMI $>200$ should be referred to a tertiary centre. Using the same criteria in premenopausal women involves referral of a larger number of benign cases. The standards for referral will undergo revisions and refinements over time. We enthusiastically await the release of better screening and diagnostic tests.

Key-words: ovarian cysts, laparoscopy, referral.

\section{JP 11_09}

Total laparoscopic hysterectomy for early-stage endometrial cancer using an intra-uterine manipulator: is it a bias for frozen section analysis?

\section{A case-control study}

M.L. Gagliardi, A. Fagotti, G. Zannoni, G. Vizzielli,

B. Costantini, V. Gallotta, G. Scambia, F. Fanfani

Division of Gynaecologic Oncology, Department

of Obstetrics and Gynaecology, Catholic University

of the Sacred Heart, Rome, Italy

Introduction: To evaluate the accuracy of frozen section diagnosis comparing the results by a laparoscopic or laparotomic approach, in order to analyze if the use of an intrauterine manipulator can alter the specimen.

Materials \& methods: Women with early stage endometrial cancer divided into a study group (114 patients), and a control group (204 patients). All patients underwent to a total hysterectomy, bilateral salpingo-oophorectomy, \pm pelvic lymphadenectomy, \pm aortic lymphadenectomy and peritoneal washing. Accuracy, over and under diagnosis of frozen section in the two groups.

Results: In the study group, all patients underwent to a total hysterectomy and bilateral salpingo-oophorectomy. Systematic bilateral pelvic lymphadenectomy was performed in 50 patients $(43.8 \%)$ and 1 patients $(0.8 \%)$ underwent to a para-aortic lymphadenectomy because had positive pelvic nodes at FS examination. In all cases $(100 \%)$ the FS diagnosis has been obtained. For the myometrial invasion, histological type and grading, the accuracy rate was of $91.2 \%, 98.2 \%$ and $93 \%$, respectively. In the control group, all patients underwent to the same surgical treatment respect to the study group. Pelvic lymphadenectomy was performed in 114 patients $(55.8 \%)$ and the median number of pelvic lymph nodes removed was 22 (range 9-58). Seven $(3.4 \%)$ patients underwent to a para-aortic lymphadenectomy because had positive pelvic nodes at FS examination. For the myometrial invasion, histological type and grading, the accuracy rate was of $85.2 \%, 98.5 \%$ and $95.1 \%$, respectively.

Discussion: Total laparoscopic hysterectomy is feasible and safe, and the intrauterine manipulator seems not to alter the frozen section.

Key-words : laparoscopy, endometrial carcinoma, frozen section

JP11_10

LARCIST: a new proposed method for the assessment of response to chemotherapy in advanced ovarian cancer patients

G. Vizzielli, F. Fanfani, B. Costantini, V. Gallotta, M.L. Gagliardi, M. Vigliotta, L. Tortorella, G. Scambia, A. Fagotti

Division of Gynaecologic Oncology, Dpt of Obstetrics and Gynaecology,Catholic University of the Sacred Heart, Rome, Italy

Summary: The aim of the study was to provide a more effective tool, based on a laparoscopic predictive value (PIV), to evaluate response to neo-adjuvant chemotherapy (NACT) in AOC patients.

Introduction: Response to chemotherapy is an important predictive factor in advanced ovarian cancer (AOC) patients. A variety of approaches in measuring response to treatment with radiological (RECIST), serological (GCIG criteria) and combined metabolic/radiological (PERCIST) criteria have been elaborated. However, they do not always correspond to a clinical/surgical management of the disease.

Materials and Methods: Sixty AOC patients primary submitted to laparoscopic surgical exploration (S-LPS) and then NACT were included in the study if they had: i) complete/partial radiological or serological response, ii) stable radiological disease in the presence of serological response, iii) progressive serological disease but stable clinical and radiological disease. All patients were submit- 
ted to a second S-LPS. Laparoscopic Response Criteria in Solid Tumors (LARCIST) were calculated as the ratio between $\triangle$-PIV (scoring gap between PIV at primary surgery and PIV at the time of IDS) and PIV at primary surgery.

Results: A statistically significant correlation between LARCIST and RECIST criteria $(\mathrm{p}=0.0001)$, with an accuracy rate of $85 \%$ was observed. On the contrary, as far as GCIG criteria are concerned, no statistical correlation was found between the two methods. As expected, response to chemotherapy is a statistically significant predictor of PFS ( $p=0.002, p=0.02$ and $p=0.4$ for LARCIST, RECIST, GCIG criteria, respectively).

Discussion: A more consistent correlation with the possibility of optimal cytoreduction has been observed for LARCIST criteria.

Key-words: laparoscopy, advanced ovarian cancer, neoadjuvant chemotherapy.

\section{JP11_11}

\section{Primary or secondary laparoscopy for staging} in patients with uterine sarcoma

B. Jahns

Department of Obstetrics and Gynaecology, Inselspital, Bern, Switzerland

Introduction: Uterine sarcomas and carcinosarcomas (CS) are rare malignancies in female patients and often diagnosed incidentally. Many patients require secondary comprehensive surgery for adequate staging. Aim of this study was to evaluate the potential role of laparoscopy in these patients.

Materials and Methods: In this retrospective chart review we analyzed the records of all patients who underwent surgery for uterine sarcoma and CS in our department between January 2002 and January 2009.

Results: We identified 39 patients with uterine sarcoma or CS who underwent primary or secondary surgery. Fifteen patients had hystological proven malignancy prior to surgery, ten patients had suspicious preoperative imaging, and 14 patients were diagnosed incidentally. Laparoscopy as comprehensive staging surgery was successfully achieved in eight patients, two at initial surgery of CS and six as secondary surgical intervention (three after initial laparotomy, three after initial laparoscopy). The number of lymph nodes did not significantly differ in the laparoscopy and the laparotomy group.

Discussion: Laparoscopy could be safely performed as primary and as secondary surgical intervention in patients with uterine sarcomas and CS, even after initial laparotomy.
More accurate diagnostic tools are required to predict patients at high risk for uterine malignancy to improve surgical management. A larger study population is needed to evaluate the feasibility of laparoscopic surgery in patients with uterine sarcoma and CS.

Key-words: carcinosarcoma, uterine sarcoma, laparoscopy.

\section{JP12_Operative Hysteroscopy}

JP12_01

Effectiveness of a polyethylene oxide-sodium carboxymethylcellulose gel in the prevention of intrauterine adhesions after hysteroscopic surgery: a pivotal study

A. Di Spiezio Sardo, M. Spinelli, M. Scognamiglio, C. Coppola, M. Guida, B. Zizolfi, P. Cirillo, C. Nappi Department of Gynaecology and Obstetrics, and Pathophysiology of Human Reproduction, University of Naples Federico II, Italy

Summary: This prospective, randomized, controlled study (Canadian Task Force classification I) assesses the efficacy of a polyethylene oxide-sodium carboxymethylcellulose gel (Intercoat, Gynecare, Ethicon, USA) in preventing the development of de-novo intrauterine adhesions (IUA) following hysteroscopic surgery. It demonstrates the Intercoat gel to reduce significantly the incidence and severity of de-novo formation of IUA after hysteroscopic surgery, improving the patency of intrauterine adhesions (IUO) at 1 month follow-up hysteroscopy.

Introduction: A prospective, randomized, controlled study (Canadian Task Force classification I) was conducted to assess the efficacy of a polyethylene oxidesodium carboxymethylcellulose gel (Intercoat, Gynecare, Ethicon, USA) in preventing the development of de-novo intrauterine adhesions (IUA) following hysteroscopic surgery, as well as to rate the patency of the Internal Uterine Ostium (IUO) at 1-month follow-up diagnostic hysteroscopy.

Materials and Methods: One hundred and ten $(n=110)$ patients diagnosed at office hysteroscopy as having single or multiple lesions suitable for surgery or resistant dysfunctional uterine bleeding requiring endometrial ablation. Patients were randomized into two different groups. Group A underwent hysteroscopic surgery plus intrauterine application of the Intercoat gel while group B underwent hysteroscopic surgery only (control group). A follow-up office hysteroscopy was performed one month after surgery to assess the rate and severity of intra-uterine adhesion 
formation and to rate the patency of the IUO after the intervention.

Results: Group A showed a significant reduction in both formation $(6 \%$ vs $22 \%$ p $<.05)$ and severity $(3 \%$ vs $25 \%$ graded as severe $\mathrm{p}<.05$ ) of de-novo IUA in comparison with the control group. Furthermore, group A showed a significant improvement in the degree of patency of the IUO ( $41.9 \%$ of cases) in comparison with diagnostic office hysteroscopies performed at the enrolment $(\mathrm{P}<.05)$. On the contrary, in group B, a worsening in the patency of the IUO was recorded in $18.2 \%$ of cases.

Discussion: The Intercoat gel reduces significantly the incidence and severity of de-novo formation of intrauterine adhesions after hysteroscopic surgery. Furthermore, it does significantly improve the patency of IUO at 1 month follow-up hysteroscopy. However, larger studies are needed to confirm our findings.

Key-words: intercoat gel, operative hysteroscopy, intrauterine adhesions.

\section{JP12 02}

\section{Hysteroscopic sterilization in IUD carriers: Department's experience \\ N. Nogueira Martins, R.M. Pinto, J. Damasceno Costa, \\ A. Pipa, F. Nogueira Martins \\ Viseu, Portugal}

Introduction: The placement of Essure ${ }^{\circledR}$ tubal microimplants in women with an intrauterine device (IUD) in place has become increasingly more frequent as this Department began to use hysteroscopy as a means to achieve definitive contraception in general. Related questions would be if such situations demand additional technical requirements, whether they would be expected to bring particular difficulties to the application of the implants or not, and if there would be differences in efficacy.

Materials and Methods: The authors made a retrospective study, where they included the 22 patients carrying an IUD who underwent Essure sterilization at their Department, in a period between December, 2006 and June, 2010. The outpatient surgical procedure and the follow-up appointment and control (three months after placement), was the same as for the non-IUD patients.

Results: In all 22 patients it was possible to place the micro-implants; in 21 cases $(95.4 \%)$ they were placed at the very first attempt and in one case $(4.6 \%)$ they were placed at the second attempt. During application, 7 of the IUDs had to be removed: 5 due to conflict of space and in 2 cases due to IUD misplacement. There were no major complications, either intra-operatively for all the patients and on the follow-up appointment for 13 patients (9 patients haven't reached the 3 months yet), where the IUDs were removed.

Conclusions: Essure ${ }^{\circledR}$ hysteroscopic sterilization remains an excellent option in IUD carriers, as it shows no significant differences in this small group when compared to standard non-IUD women. In 7 patients $(33.3 \%)$ there was the need to remove the IUD during the application of the micro-implants, which subsequently warrants proper prior information to the patient in that sense before placement. The results were limited by a rather small size of our sample and, therefore, larger series seem to be advisable. Nevertheless, all the results up to this point at our Department are similar to the ones obtained for the general target population of this technique.

Key-words: hysteroscopy, contraception, IUD.

\section{JP15_Single Access Surgery}

\section{JP 1501}

Feasibility and efficacy of SPAL for adnexal pathology G. Maricosu, L. Mereu, L. Mencaglia, G.B. Melis, S. Angioni Division of Gynaecology, Obstetrics and Pathophysiology of Human Reproduction, University of Cagliari and Division of Gynaecology, Villanova Hospital of Florence, Italy

Objective: To present our initial experience using single access laparoscopic surgery for the treatment of benign adnexal pathologies.

Methods: Thirty patients with benign adnexal pathologies underwent salpingo-oophorectomy $(n=15)$, ovarian cyst enucleation $(n=10)$, or salpingectomy $(n=5)$ using a laparoendoscopic single site approach with a new multiport reusable trocar (S-Portal X-Cone Storz, Tuttlingen) and flexible and curved as well as standard laparoscopic instruments.

Results: Conversion to a multi-access standard laparoscopic technique was not required in any patient and no intraoperative complications were observed. Postoperatively, one umbilical scar infection was detected. Mean operative time was 42 minutes.

Discussion: Laparoscopic single site surgery for adnexal pathologies is feasible, safe, and effective, and has good results for cosmetic appearance and postoperative pain. Use of specialized instruments and standardization of the technique affect surgical ergonomy and operating time.

Key-words: SPAL, cystectomy, adnexectomy. 


\section{JP 15_02}

Total Laparo-Endoscopic Single Site Surgery (LESS). Hysterectomy in low-risk early endometrial cancer: technique and preliminary results

C. Rossitto, A. Fagotti, V. Gallotta, M.L. Gagliardi,

S. Gueli Alletti, B. Costantini, G. Vizzielli, G. Scambia,

F. Fanfani

Division of Gynaecologic Oncology, Dpt Obstetrics and Gynaecology, Catholic University of the Sacred Heart, Rome, Italy

Summary: LESS could represent a safe and adequate surgical option for hysterectomy in early-stage endometrial cancer patients Introduction: To present our initial experience with laparo-endoscopic single site surgery (LESS) in the surgical treatment of early-stage endometrial cancer patients.

Materials and Methods: Between July 1 and February 2010, 15 consecutive low-risk early endometrial cancer referred to the Division of Gynecologic Oncology of the Catholic University of the sacred Heart of Rome were enrolled in the study. All patients underwent total LESS hysterectomy. Intra- and early post-operative outcomes were collected.

Results: Median age was 57 (42-68) and median BMI was 24 (21-30). Median operative time was 120 minutes (range 85-155) and median estimated blood loss $20 \mathrm{ml}$ (range 10180). Larger skin and fascial incision required by LESS approach was $2.5 \mathrm{~cm}$ (median $2.2 \mathrm{~cm}$; range 2.0-2.5). No laparoscopic/laparotomic conversion was registered, and no additional port insertion was necessary. Median ileus was 16 hours (range 12-20) and median time to discharge was 1 (range 1-2). All patient conveyed complete satisfaction towards cosmetic result and post-operative pain control.

Discussion: LESS can represent a safe and adequate surgical option for hysterectomy in early-stage endometrial cancer patients, with the potential to further decrease invasiveness of the conventional laparoscopic approach. Key-words: endometrial carcinoma, single-site surgery.

\section{JP16_Teaching \& Training}

\section{JP 16_01}

Trainees experience of laparoscopic management of tubal pregnancy in the UK

R. Bharathan, S. Meritte, H. Ahmed

Medway Maritime Hospital, Kent, United Kingdom

High quality evidence recommends laparoscopic management of tubal pregnancy. The last national survey in the
U.K published in 2000 found only 35\% of cases was performed laparoscopically; recent single institution figures exceed $90 \%$. In a recent study of UK trainees $53.3 \%$ said they were being trained in this procedure. Factors that impede optimal training include the working time directive, lack of suitable trainers and organisation of resources. Since competency in this procedure is mandatory for certification in U.K, our survey explores trainees' experience. A national postal survey of U.K residents was performed in 2009. The response rate was $31.6 \%$, amongst 645 residents. The median number of procedures where they performed some component of the procedure was 4 , and where they performed most of the procedure was $3.75 \%$ of trainees had performed at least most of 1 procedure. $43 \%$ practiced salpingotomy. $50 \%$ preferred diathermy excision rather than endoloop; $3 \%$ used both techniques equally. $47 \%$ of trainees had attended an endoscopy course within 2 years. 30\% reported they had access to a simulator. On a 5 point Likert scale, the mean training satisfaction score was 3.0 and the mean score regarding anticipation of achieving competency was 3.8 . In this survey $94 \%$ of trainees had performed at least some component of the surgery. The learning curve of salpingotomy is steeper than that for salpingectomy. Endoloop excision is quicker surgery, with less analgesia use and shorter hospital stay compared to diathermy; these are important training considerations. There is good evidence to support courses and simulators in aiding the acquisition and retention of skills. These factors must be addressed within a formal curriculum.

Key-words: tubal pregnancy, laparoscopic surgery, training.

\section{JP 16_02}

\section{Comparison of textbook versus educational video for training in office operative hysteroscopy: a randomized, controlled trial}

A. Di Spiezio Sardo, M. Spinelli, L.M. Sosa Fernandez, M. Scognamiglio, M. Lisbino, P. Cirillo, M. Guida, C. Nappi

Department of Gynaecology and Obstetrics, and Pathophysiology of Human Reproduction, University of Naples Federico II, Italy

Summary: A prospective, randomized, controlled trial (Canadian Task Force classification I) was performed in order to compare the effectiveness of textbook versus educational video for training in office operative hysteroscopy. It demonstrates the use of video as a teaching modality for superior conceptual understanding, as a component of hysteroscopic performance. 
Introduction: Video seems intuitively to be advantageous over traditional text as an educational tool in conceptually based procedures such as laparoscopy. However, this has never been tested or validated.

Materials and Methods: Thirty trainees, confident in performing office diagnostic hysteroscopy but unskilled in outpatient operative procedures. All participants were trained on how to perform simple operative procedures in the office setting, being randomized into one of two teaching modalities: using a textbook supported by iconographic materials (Group A: 15) or watching an audio-video tutorial of comparable content (Group B: 15). Time for reviewing the educational material could be tailored to individual needs. Following the theoretical sessions, throughout a two weeks period of in-vivo office training, each participant was asked to perform five operative procedures including: eye-target endometrial biopsies, adhesiolysis and small cervical and endometrial polypectomy under the close supervision of an experienced operator. Both 5Fr mechanical instruments and bipolar electrodes could be used. The operative procedure was considered failed whether the time required to accomplish it was over the set deadline and/or whether the performance of the trainee was judged as inadequate by the supervisor.

Results: Time to review the educational material was significantly higher in Group A $(p<.001)$. The number of trainees who showed adequate insight ( 9 vs $15 ; \mathrm{p}=.006$ ) and who were able to successfully perform all five operative procedures ( 6 vs $13 ; \mathrm{p}=.008$ ) was significantly higher in Group B. No significant difference in the mean time required to successfully complete the procedures was reported between the two groups.

Discussion: Our study supports the use of educational videos over the conventional textbook training for office operative hysteroscopy in that it seems to lead to a greater insight of the procedure as well as a better performance.

Key-words: training, hysteroscopy, educational video.

\section{JP18 Urogynaecology}

\section{JP 1801}

Complications of mesh use in pelvic organ prolapse surgery-retrospective multicentre study of 677 patients V.I. Krasnopolsky, A.A. Popov, K.N. Abramyan, T.N. Manannikova, M.R. Ramazanov, M.A. Chechneva, A.A. Fedorov, I. V. Krasnopolskaya, B.A. Slobodyanyuk, O.V. Machanskite

Moscow Regional Scientific Research Institute, Russia

Introduction and Hypothesis: We describe complications associated with the use of transvaginal mesh for treatment of pelvic organ prolapse.

Study design: Since January 2005 till March 2010, 677 patients were included in a retrospective multicentre study. All of them had III-IV stage POP-Q and were underwent surgical management by the use of Prolift ${ }^{\circledR}$ system. Surgical protocol was standardized according to an original technique.

Results: In total, 677 operations were done: 303-Prolift anterior, 51-Prolift posterior, 232-Prolift total, 91-Prolift anterior and posterior. Results were analyzed, summarized and divided into three groups. 1. Intra-operative complications occurred in 37 cases: urethral injuries - $2(0,3 \%)$, bladder injuries-10 $(1,5 \%)$, rectal injuries - $5(0,7 \%)$, haemorrhages $(>500 \mathrm{ml})-19(2,8 \%)$. 2. Post-operative complications occurred in 72 cases: hyperthermia over $38^{\circ} \mathrm{C}-11(1,6 \%)$, voiding dysfunction - $18(2,7 \%)$, vaginal haematomas -43 (6,4\%). 3. Mesh-related complications reported in 62 cases: vaginal erosions (managed surgically) - $32(4,7 \%)$, bladder erosion-1 $(0,1 \%)$, perineal abscess (required complete mesh removal) - $4(0,6 \%)$, mesh-shrinkage with vaginal contraction-8 $(1,2 \%)$, pain-18 $(2,7 \%)$.

Conclusion: Using transvaginal mesh in pelvic reconstruction should not be a routine procedure, because of the complications occur in $25 \%$, some of them are extremely severe, can be life threatening and required highly specialized management.

Key-words: complication, prolift, POP. 